\title{
Cosmological attractors in massive gravity
}

\author{
S. L. Dubovsky, ${ }^{1,3}$ P. G. Tinyakov, ${ }^{2,3}$ and I. I. Tkachev ${ }^{1,3}$ \\ ${ }^{1}$ Department of Physics, CERN Theory Division, CH-1211 Geneva 23, Switzerland \\ ${ }^{2}$ Service de Physique Théorique, Université Libre de Bruxelles, CP225, blv. du Triomphe, B-1050 Bruxelles, Belgium \\ ${ }^{3}$ Institute for Nuclear Research of the Russian Academy of Sciences, 60th October Anniversary Prospect, 7a, 117312 Moscow, Russia
}

(Received 24 May 2005; published 13 October 2005)

\begin{abstract}
We study Lorentz-violating models of massive gravity which preserve rotations and are invariant under time-dependent shifts of the spatial coordinates. In the linear approximation the Newtonian potential in these models has an extra confining term proportional to the distance from the source. We argue that during cosmological expansion the Universe may be driven to an attractor point with larger symmetry which includes particular simultaneous dilatations of time and space coordinates. The confining term in the potential vanishes as one approaches the attractor. In the vicinity of the attractor the extra contribution is present in the Friedmann equation which, in a certain range of parameters, gives rise to the cosmic acceleration.
\end{abstract}

DOI: 10.1103/PhysRevD.72.084011

\section{INTRODUCTION}

Recently it has been realized [1-3] that models of modified gravity which contain Lorentz-violating graviton mass terms may avoid all known problems of massive gravity such as the van Dam-Veltman-Zakharov (vDVZ) discontinuity [4,5], ghost instabilities [6] and strong quantum effects at the unacceptably low energy scale [7]. ${ }^{1}$ In the most general sense by massive gravity we understand any theory described by the following action:

$$
S=-M_{\mathrm{Pl}}^{2} \int d^{4} x \sqrt{-g} R+\int d^{4} x \sqrt{-g} F,
$$

where the first term is a usual Einstein-Hilbert term and $F$ is, generally speaking, an arbitrary (noncovariant) function of metric components and their derivatives. Matter fields are assumed to be covariantly coupled to the metric. A systematic study of the rotationally invariant theories of massive gravity was performed in Ref. [3]. A particularly interesting class of models found there is characterized by a residual reparametrization symmetry

$$
x^{i} \rightarrow x^{i}+\xi^{i}(t)
$$

where $x^{i}$ are the spatial coordinates. This class of models is singled out by the following two properties. First, in the vicinity of the Minkowski background these models represent consistent low-energy effective theories valid up to the energy scale $\Lambda \sim \sqrt{m M_{\mathrm{Pl}}}$, where $m$ is a graviton mass. The absence of ghosts and classical instabilities is ensured by the symmetry requirements alone without need for any extra fine-tuning. The second important property is that already the lowest-dimension operators (graviton mass terms) lead to the modification of gravity, in particular, gravitational waves are massive. An example of a theory

\footnotetext{
${ }^{1}$ It is worth mentioning that another intriguing route to solve these problems may be to take into account the effects of local curvature [8-10].
}

PACS numbers: 04.50.+h, 04.30. $-\mathrm{w}, 95.35 .+\mathrm{d}, 98.80 .-\mathrm{k}$

which shares the first but not the second property is the ghost condensate model [1] which, at the lowest derivative level, is equivalent to the gauge-fixed Einstein theory. Another class of theories with this property is discussed in Refs. [11,12]. In what follows by massive gravity we understand gravity theories (1) obeying symmetry (2).

The phenomenological consequences of the massive gravity models obeying symmetry (2) were first studied in Ref. [13]. One of the unexpected properties found there is that massive gravitational waves may coexist with the long-range potential between the sources. In general, the gravitational potential in these models contains an extra "confining" piece which grows linearly with the distance from the source,

$$
\Phi=G_{N} M\left(-\frac{1}{r}+\mu^{2} r\right),
$$

where $\mu^{2}$ is a combination of the graviton masses (see Sec. II) proportional to their overall scale. The analysis of Ref. [13] was focused mainly on the case $\mu^{2}=0$ when the additional dilatation symmetry ensures that the long-range potential is identical to that of the Einstein theory. This relaxes the constraints coming from the Solar system and Cavendish-type experiments, and opens up a possibility for the graviton mass to be as large as $\sim\left(10^{-15} \mathrm{~cm}\right)^{-1}$ without contradiction to the existing experimental data. The relic gravitational waves produced at inflation may constitute today the cold dark matter in the Universe and would give a unique monochromatic signal in the gravitational wave detectors [13].

The purpose of the present paper is to study cosmological solutions in the massive gravity. We address the question of whether such solutions are phenomenologically acceptable, and what are their generic properties. We do not assume that parameters are tuned so that $\mu^{2}=0$ from the very beginning. It turns out, however, that for a large class of functions $F$ in Eq. (1) the cosmological evolution naturally drives the system to the point $\mu^{2}=0$ where there 
are no corrections to the Newtonian potential. In other words, this point is an attractor in the solution space (it corresponds to the restoration of an additional dilatation symmetry). At this point the graviton mass has a finite nonzero value, while the modification of the Friedmann equation has a form of an extra term which behaves like a dark energy with the equation of state depending on the parameters of the model (properties of the function $F$ ).

The paper is organized as follows. We begin in Sec. II by analyzing linear perturbations about the flat background and, in particular, derive Eq. (3). In Sec. III we study general properties of the cosmological solutions in massive gravity. We then consider in Sec. IV the stability of the curved solutions against perturbations of high momenta (the Boulware-Deser instability) and argue that the stability is achieved without fine-tuning of parameters at least for backgrounds close to the Minkowski space. In particular, cosmological solutions found in Sec. III are stable in the vicinity of the attractor point. In the concluding Sec. V we discuss possible phenomenological applications and some future directions in the studies of the massive gravity.

\section{LINEARIZED THEORY NEAR MINKOWSKI BACKGROUND}

As has been argued in Ref. [3], a convenient way to describe the Lorentz-violating models of massive gravity is to introduce the set of four scalar "Goldstone" fields $\phi^{0}$, $\phi^{i}$, which have a particular derivative couplings to gravity. In terms of the metric and the Goldstone fields, the action has a generally covariant form. The spontaneous breaking of the covariance is achieved by assuming nonzero vacuum expectation values of the derivatives of the Goldstone fields. The Goldstone fields can be eliminated from the action by a suitable coordinate transformation; in such a "unitary gauge" the action only depends on the metric components.

A class of Lorentz-violating gravity models which possess the symmetry (2) and rotational invariance is represented by the action

$$
S=\int d^{4} x \sqrt{-g}\left[-M_{\mathrm{Pl}}^{2} R+\Lambda^{4} F\left(X, W^{i j}, \ldots\right)\right],
$$

where

$$
\begin{gathered}
X=g^{\mu \nu} \partial_{\mu} \phi^{0} \partial_{\nu} \phi^{0} \\
W^{i j}=g^{\mu \nu} \partial_{\mu} \phi^{i} \partial_{\nu} \phi^{j}-\frac{g^{\mu \nu} \partial_{\mu} \phi^{0} \partial_{\nu} \phi^{i} \cdot g^{\lambda \rho} \partial_{\lambda} \phi^{0} \partial_{\rho} \phi^{j}}{X},
\end{gathered}
$$

dots stand for higher-derivative terms, and $\Lambda$ is a parameter which determines the cutoff scale of the theory. The indices $i, j$ are converted using $\delta_{i j}$. Low-energy modification of gravity takes place at the scale $m \sim \Lambda^{2} / M_{\mathrm{Pl}}$. The function $F$ is arbitrary apart from the constraints following from the requirement that the model is free of ghosts and strongcoupling problems [3]; we assume that it depends on a single scale $\Lambda$. The coefficient in front of the EinsteinHilbert action is chosen for convenience.

We assume that the model (4) possesses the solution which corresponds to the Minkowski space,

$$
g_{\mu \nu}=\eta_{\mu \nu}, \quad \phi^{0}=\alpha \Lambda^{2} t, \quad \phi^{i}=\beta \Lambda^{2} x^{i} .
$$

Here $\alpha$ and $\beta$ are some constants which have to be chosen in such a way that the energy-momentum tensor of the Goldstone fields is zero. This requirement reduces to two Eqs. (A9) and (A10), shown in the Appendix A. Consequently, this choice is possible for a generic function $F$.

Our current goal is to study linear perturbations about the vacuum (6). It is convenient to work in the "unitary gauge" where the Goldstone fields are set to their vacuum values (6). In this gauge the remaining perturbations are those of the metric, $\delta g_{\mu \nu}$,

$$
g_{\mu \nu}=\eta_{\mu \nu}+\delta g_{\mu \nu} .
$$

Following the notations of Ref. [14], we parametrize $\delta g_{\mu \nu}$ as follows:

$$
\begin{gathered}
\delta g_{00}=2 \varphi ; \quad \delta g_{0 i}=S_{i}-\partial_{i} B ; \\
\delta g_{i j}=-h_{i j}-\partial_{i} F_{j}-\partial_{j} F_{i}+2\left(\psi \delta_{i j}-\partial_{i} \partial_{j} E\right),
\end{gathered}
$$

where $h_{i j}$ are the transverse and traceless tensor perturbations, $S_{i}$ and $F_{i}$ are the transverse vector perturbations, while $\varphi, \psi, B$ and $E$ are the scalar perturbations. The potential $\varphi$ is not to be confused with the Goldstone fields $\phi_{0}$ and $\phi_{i}$.

The quadratic Lagrangian for perturbations has the form

$$
L=L_{E H}+L_{m}+L_{s},
$$

where the three contributions are the Einstein-Hilbert term, the mass term and the source term, respectively. The quadratic part of the Einstein-Hilbert Lagrangian is

$$
\begin{aligned}
L_{E H}= & M_{\mathrm{Pl}}^{2}\left\{-\frac{1}{4} h_{i j}\left(\partial_{0}^{2}-\partial_{i}^{2}\right) h_{i j}\right. \\
& -\frac{1}{2}\left(S_{i}+\partial_{0} F_{i}\right) \partial_{j}^{2}\left(S_{i}+\partial_{0} F_{i}\right) \\
& \left.+4\left(\varphi+\partial_{0} B-\partial_{0}^{2} E\right) \partial_{i}^{2} \psi+6 \psi \partial_{0}^{2} \psi-2 \psi \partial_{i}^{2} \psi\right\} .
\end{aligned}
$$

The mass term originates from the second term in Eq. (4). We parametrize the mass parameters according to the notations of Ref. [2],

$$
\begin{aligned}
L_{m}= & \frac{M_{\mathrm{Pl}}^{2}}{4}\left\{m_{0}^{2} \delta g_{00}^{2}+2 m_{1}^{2} \delta g_{0 i}^{2}-m_{2}^{2} \delta g_{i j}^{2}+m_{3}^{2} \delta g_{i i}^{2}\right. \\
& \left.-2 m_{4}^{2} \delta g_{00} \delta g_{i i}\right\} .
\end{aligned}
$$

The contribution proportional to $\delta g_{0 i}^{2}$ is absent in our 
model $\left(m_{1}^{2}=0\right)$. This is guaranteed by the symmetry (2). In terms of tensor, vector and scalar perturbations these mass terms read

$$
\begin{aligned}
& M_{\mathrm{Pl}}^{2}\left\{-\frac{1}{4} m_{2}^{2} h_{i j}^{2}-\frac{1}{2} m_{2}^{2}\left(\partial_{i} F_{j}\right)^{2}+m_{0}^{2} \varphi^{2}+\left(m_{3}^{2}-m_{2}^{2}\right)\left(\partial_{i}^{2} E\right)^{2}\right. \\
& \quad-2\left(3 m_{3}^{2}-m_{2}^{2}\right) \psi \partial_{i}^{2} E+3\left(3 m_{3}^{2}-m_{2}^{2}\right) \psi^{2}+2 m_{4}^{2} \varphi \partial_{i}^{2} E \\
& \left.\quad-6 m_{4}^{2} \varphi \psi\right\} .
\end{aligned}
$$

The masses $m_{i}^{2}$ are expressed in terms of the first and second derivatives of the function $F$, the parameter $\Lambda$ and the Planck mass. The overall scale of masses is set by the ratio $\Lambda^{2} / M_{\mathrm{Pl}}$. The explicit expressions are given in the Appendix A.

To probe the linear response of the system we add the source $T_{\mu \nu}$ which is assumed to be conserved, $\partial^{\mu} T_{\mu \nu}=0$. The corresponding contribution to the Lagrangian can be written as

$$
\begin{aligned}
L_{s}= & -T_{00}\left(\varphi+\partial_{0} B-\partial_{0}^{2} E\right)-T_{i i} \psi+\left(S_{i}+\partial_{0} F_{i}\right) T_{0 i} \\
& +\frac{1}{2} h_{i j} T_{i j} .
\end{aligned}
$$

All combinations coupled to the components of $T_{\mu \nu}$ are gauge invariant. The one multiplying $T_{00}$,

$$
\Phi \equiv \varphi+\partial_{0} B-\partial_{0}^{2} E
$$

plays the role of the Newtonian potential in the nonrelativistic limit.

(a) Tensor sector. - In the tensor sector, only the transverse traceless perturbations $h_{i j}$ are present. Their field equation is that of a massive field with the mass $m_{2}$, in agreement with Ref. [2]. Thus, there are two massive spin 2 propagating degrees of freedom.

(b) Vector sector. - In the vector sector, the field equations read

$$
\begin{gathered}
-\partial_{j}^{2}\left(S_{i}+\partial_{0} F_{i}\right)=-T_{0 i}, \\
\partial_{0} \partial_{j}^{2}\left(S_{i}+\partial_{0} F_{i}\right)+m_{2}^{2} \partial_{j}^{2} F_{i}=\partial_{0} T_{0 i} .
\end{gathered}
$$

Taking the time derivative of Eq. (12) and adding it to Eq. (13) gives

$$
F_{i}=0,
$$

provided that $m_{2}^{2} \neq 0$. Thus, the vector sector of our model behaves in the same way as in the Einstein theory in the gauge $F_{i}=0$. There are no propagating vector perturbations and gravity is not modified in the vector sector unless one takes into account nonlinear effects or higher-derivative terms.

(c) Scalar sector.-The field equations for scalar perturbations are

$$
\begin{gathered}
2 \partial_{i}^{2} \psi+m_{0}^{2} \varphi+m_{4}^{2} \partial_{i}^{2} E-3 m_{4}^{2} \psi=\frac{T_{00}}{2 M_{\mathrm{Pl}}^{2}}, \\
2 \partial_{i}^{2} \Phi-2 \partial_{i}^{2} \psi+6 \partial_{0}^{2} \psi-\left(3 m_{3}^{2}-m_{2}^{2}\right) \partial_{i}^{2} E \\
+3\left(3 m_{3}^{2}-m_{2}^{2}\right) \psi-3 m_{4}^{2} \varphi=\frac{T_{i i}}{2 M_{\mathrm{Pl}}^{2}}, \\
-2 \partial_{i}^{2} \partial_{0}^{2} \psi+\left(m_{3}^{2}-m_{2}^{2}\right) \partial_{i}^{4} E-\left(3 m_{3}^{2}-m_{2}^{2}\right) \partial_{i}^{2} \psi \\
+m_{4}^{2} \partial_{i}^{2} \varphi=-\frac{\partial_{0}^{2} T_{00}}{2 M_{\mathrm{Pl}}^{2}}, \\
2 \partial_{i}^{2} \partial_{0} \psi=\frac{\partial_{0} T_{00}}{2 M_{\mathrm{Pl}}^{2}} .
\end{gathered}
$$

Equation (17) implies

$$
\psi=\frac{1}{\partial_{i}^{2}} \frac{T_{00}}{4 M_{\mathrm{Pl}}^{2}}+\psi_{0}\left(x^{i}\right),
$$

where $\psi_{0}\left(x^{i}\right)$ is some time-independent function. From Eqs. (14) and (16) one finds

$$
\begin{gathered}
\varphi=\frac{2 m_{2}^{2} m_{4}^{2}}{\Delta} \psi+\frac{2\left(m_{3}^{2}-m_{2}^{2}\right)}{\Delta} \partial_{i}^{2} \psi_{0}, \\
\partial_{i}^{2} E=\left(3-\frac{2 m_{0}^{2} m_{2}^{2}}{\Delta}\right) \psi-\frac{2 m_{4}^{2}}{\Delta} \partial_{i}^{2} \psi_{0},
\end{gathered}
$$

where

$$
\Delta=m_{4}^{4}-m_{0}^{2}\left(m_{3}^{2}-m_{2}^{2}\right) .
$$

Finally, substituting Eqs. (18)-(20) into Eq. (15) one finds the gauge-invariant potential $\Phi$,

$$
\begin{aligned}
\Phi= & \frac{1}{\partial_{i}^{2}} \frac{T_{00}+T_{i i}}{4 M_{\mathrm{Pl}}^{2}}-3 \frac{\partial_{0}^{2}}{\partial_{i}^{4}} \frac{T_{00}}{4 M_{\mathrm{Pl}}^{2}} \\
& +\left(3-\frac{2 m_{0}^{2} m_{2}^{2}}{\Delta}\right) \frac{m_{2}^{2}}{\partial_{i}^{2}}\left(\frac{1}{\partial_{i}^{2}} \frac{T_{00}}{4 M_{\mathrm{Pl}}^{2}}+\psi_{0}\right) \\
& +\left(1-\frac{2 m_{2}^{2} m_{4}^{2}}{\Delta}\right) \psi_{0},
\end{aligned}
$$

where we presented explicitly the dependence on $\psi_{0}$ and $T_{\mu \nu}$. The first two terms on the right-hand side (rhs) of Eq. (2) are the standard contributions in the Einstein theory, the first becoming the Newtonian potential in the nonrelativistic limit. Thus, barring the $\psi_{0}$-dependent terms, the gauge-invariant potentials $\Phi$ and $\psi$ in our model differ from their analogs in the Einstein theory $\Phi_{E}$ and $\psi_{E}$ by the mass-dependent third term on the rhs of Eq. (21),

$$
\psi=\psi_{E}, \quad \Phi=\Phi_{E}+\left(3-\frac{2 m_{0}^{2} m_{2}^{2}}{\Delta}\right) \frac{m_{2}^{2}}{\partial_{i}^{4}} \frac{T_{00}}{4 M_{\mathrm{Pl}}^{2}} .
$$

This term vanishes if all masses uniformly go to zero, which implies the absence of the vDVZ discontinuity. Equation (22) is the result presented in Ref. [13]. For a 
static source, Eq. (22) leads to the modification of the Newtonian potential of a point mass $M$ as shown in Eq. (3) with

$$
\mu^{2}=-\frac{1}{2} m_{2}^{2}\left(3-\frac{2 m_{0}^{2} m_{2}^{2}}{\Delta}\right)
$$

This indicates the breakdown of perturbation theory at distances $r \gtrsim 1 /\left(G_{N} M \mu^{2}\right)$. Note, that the modification of the Newtonian potential is absent if $3 \Delta=2 m_{0}^{2} m_{2}^{2}$ (and $\Delta \neq 0$ ). We will see in Sec. III that this happens in the vicinity of the cosmological attractor, i.e. at late times of the cosmological evolution.

The freedom of choosing the time-independent function $\psi_{0}(x)$, which enters the above gravitational potentials, indicates the presence of the scalar mode with the dispersion relation $^{2} \omega^{2}=0$ [3] [cf. also Sec. IV, Eq. (45) in the limit $\left.m_{1}^{2} \rightarrow 0\right]$. This mode is an analogue of the ghost condensate mode [1] and becomes dynamical with the account of higher-derivative terms in the action (4), acquiring the dispersion relation $\omega^{2} \propto p^{4}$ (so that $\psi_{0}$ becomes a slowly varying function of time). The value of $\psi_{0}$ is fixed by the initial conditions. In the linear regime, the nonzero value $\psi_{0}$ would mean the presence of the incoming "ghost condensate wave." So, a physically reasonable choice of $\psi_{0}$ is $\psi_{0}\left(x^{i}\right)=0$. We will discuss in more detail a possible role of this mode in the concluding Sec. V.

Note that the kinetic term of the ghost condensate mode is proportional to the combination $\Delta$ [3] (see also Sec. IV). Therefore, in general this combination should be nonzero (positive), in agreement with Eqs. (19)-(22). It may happen that in some special cases one can obtain a healthy theory even if $\Delta=0$. An interesting possibility suggested recently in Ref. [15] is to impose an additional condition $m_{4}^{2}=m_{0}^{2}=0$. Then the "ghost condensate" mode does not appear in the linearized theory at the lowest derivative level in flat background. It acquires both kinetic and gradient term at a higher-derivative level, so that an additional symmetry $t \rightarrow t+\xi^{0}(t)$ is needed to prevent this mode from being a ghost. The gravitational potentials are nonsingular in this case despite $\Delta=0$ and have the same structure as our Eq. (22). As we discuss in Sec. IV, the stability of this model requires further study and is more subtle than in the case $\Delta \neq 0$ because of new propagating modes that appear in curved backgrounds.

\section{COSMOLOGICAL SOLUTIONS}

Let us discuss flat cosmological solutions in the theory defined by the action (4). The flat cosmological ansatz is

$$
\begin{gathered}
d s^{2}=d t^{2}-a^{2}(t) d x_{i}^{2}, \\
\phi^{0}=\phi(t), \quad \phi^{i}=\Lambda^{2} x^{i} .
\end{gathered}
$$

\footnotetext{
${ }^{2}$ In what follows $\omega$ denotes frequency and $p$ denotes the absolute value of the spatial momentum.
}

For this ansatz $W^{i j}=-a^{-2} \delta^{i j}$, so the function $F$ in (4) depends only on $X$ and $a, F=F(X, a)$. The Einstein equations reduce to the Friedmann equation (see Appendix A),

$$
\begin{aligned}
\left(\frac{\dot{a}}{a}\right)^{2} & =\frac{1}{6 M_{\mathrm{Pl}}^{2}}\left\{\rho_{m}+2 \Lambda^{4} X F_{X}-\Lambda^{4} F\right\} \\
& \equiv \frac{1}{6 M_{\mathrm{Pl}}^{2}}\left\{\rho_{m}+\rho_{1}+\rho_{2}\right\},
\end{aligned}
$$

where $\rho_{m}$ is the energy density of ordinary matter not including Goldstone fields, and the field equation for $\phi^{0}$,

$$
\partial_{t}\left(a^{3} \sqrt{X} F_{X}\right)=0 .
$$

It is straightforward to solve this system of equations for any given function $F(X, a)$. After the integration, Eq. (27) gives an algebraic equation which determines $X$ as a function of the scale factor $a$. The dependence $X(a)$ as found from Eq. (27) determines the behavior of the Goldstone energy density $\rho_{1}+\rho_{2}$ as a function of $a$. This makes Eq. (26) a closed equation for the scale factor $a(t)$.

From the point of view of cosmological applications, of particular interest are solutions where the scale factor $a(t)$ goes to infinity at late times. Since the graviton masses are linear combinations of the function $F(X, a)$ and its derivatives, one may wonder whether they remain finite or go to zero in this limit, and whether the effective-theory description remains valid. Indeed, Eq. (27) implies that at late times either $X$ or $F_{X}$ go to zero. If $X \rightarrow 0$, then the expressions given in the Appendix A suggest that the graviton masses go to zero as well. This may lower the cutoff scale of the effective theory. Similarly, some of the masses apparently vanish if $X$ goes to a finite value $X_{0}$ such that $F_{X}\left(X_{0}, a\right) \rightarrow 0$. If $X$ goes to infinity, this questions the validity of the low-energy effective theory by itself.

Let us show that, in spite of the naive expectations, for a wide class of functions $F$ there exist solutions for which graviton masses are finite in the limit $a \rightarrow \infty$ and the effective-theory description remains valid. Assume that $X(a)$ asymptotes to some power of $a$ at large $a$. This is not a very restrictive assumption - for instance, it is satisfied for any algebraic function $F(X, a)$. Then there exists such $\gamma$ that the combination $X^{\gamma} / a^{2}$ goes to a nonzero constant as $a \rightarrow \infty$. Equation (27) implies that $X F_{X}=$ const $\cdot \sqrt{X} / a^{3}$; this determines the dependence of the energy component $\rho_{1}$ on the scale factor,

$$
\rho_{1}=\text { const } \frac{1}{a^{3-1 / \gamma}} \text {. }
$$

This relation generalizes the behavior found in the ghost condensate models where the energy density of the ghost condensate scales like $1 / a^{3}$ (in our model the latter behavior is recovered at $\gamma \rightarrow \infty$ ). 
For $\gamma>1 / 3$ the energy density $\rho_{1}$ behaves like the dark energy component with the negative pressure. Its equation of state varies between that of the cold dark matter, $w=0$ (for $\gamma=+\infty$ ), and that of the cosmological constant, $w=-1$ (for $\gamma=\frac{1}{3}$ ). For $0<\gamma<1 / 3$ the term $\rho_{1}$ grows with $a$. It corresponds to the energy density component with a highly negative equation of state, $w<-1$. Without fine-tuning this contribution cannot be canceled by the term $\rho_{2}$, so that the Hubble rate diverges as $a \rightarrow \infty$ leading to the breakdown of the low-energy effective theory and suggesting the presence of rapid instabilities. In what follows we assume that $\gamma$ does not belong to this range. For $\gamma<0$ the energy density $\rho_{1}$ corresponds to a fluid with a positive pressure.

In order to see that the graviton masses remain finite and the effective field theory description is valid in the limit $a \rightarrow \infty$, it is convenient to replace $X$ by a new variable $Z=$ $X^{\gamma} / a^{2}$. The function $F(X, a)$ becomes the function of $Z$ and $a, \tilde{F}(Z, a)=F\left(Z^{1 / \gamma} a^{2 / \gamma}, a\right)$. Note that it satisfies the relation $\gamma Z \tilde{F}_{Z}=X F_{X}$, where $\tilde{F}_{Z}=\partial \tilde{F} / \partial Z$. In these notations Eq. (27) reads

$$
\gamma a^{3-(1 / \gamma)} Z^{1-(1 / 2 \gamma)} \tilde{F}_{Z}(Z, a)=A,
$$

where $A$ is an integration constant. This equation determines $Z$ as a function of $a$. By construction, this dependence is such that $Z(a \rightarrow \infty)=Z_{0}$, where $Z_{0}$ is some constant.

If one assumes further that the function $\tilde{F}(Z, a)$ is regular at $a \rightarrow \infty$, then at late times one has

$$
F(X, a)=\tilde{F}(Z, a) \rightarrow F_{0}(Z) .
$$

In terms of the original variables this means that in the limit $a \rightarrow \infty$ the function $F\left(X, W^{i j}\right)$ depends only on the combination $X^{\gamma} W^{i j}$. This corresponds to the following dilatation symmetry of the Goldstone action:

$$
\phi_{0} \rightarrow \lambda \phi_{0}, \quad \phi_{i} \rightarrow \lambda^{-\gamma} \phi_{i} .
$$

In this case one has

$$
\rho_{2}=-\Lambda^{4} F_{0}\left(Z_{0}\right),
$$

which behaves like a cosmological constant [assuming $F_{0}\left(Z_{0}\right) \neq 0$ ]. Likewise, at $a \rightarrow \infty$ the masses given by Eqs. (A13)-(A17) become functions of $Z_{0}$ and in general remain finite.

In models with this kind of behavior of $X(a)$ the effective field theory description remains valid even at $X \gg \Lambda^{4}$ provided the value of $Z$ is small. This is guaranteed by the dilatation symmetry (31) which relates configurations with different values of $X$. Thus, there exists a wide class of functions $F$ for which infinitely expanding cosmological solutions are compatible with constant graviton masses and allow for the effective field theory description.

Our assumptions about the function $F$ which imply the existence of the attractors can be summarized in the following expansion:

$$
F(Z, W)=F_{0}(Z)+\sum_{\nu>0} \epsilon^{\nu} W^{\nu} F_{\nu}(Z)
$$

where $\nu$ takes positive (not necessarily integer) values, $F_{\nu}(Z)$ are some regular functions of $Z$ (for shortness we have suppressed the indices $i, j$ ) and $\epsilon$ is a formal expansion parameter. Equation (29) implies that an attractor point $Z_{0}$ is determined by the condition

$$
F_{0}^{\prime}\left(Z_{0}\right)=0,
$$

where prime denotes $d / d Z$. Note that the expansion (32) does not need to hold for arbitrary values of $Z$ and $W$; it is sufficient if it is satisfied in some finite region around the attractor point. Equation (32) provides a straightforward way to generate simple functions $F$ depending on both $X$ and $W$ and exhibiting the attractor behavior. A simple example of such a function is $F=\left(Z-Z_{0}\right)^{2}+W$.

One may wonder whether the class of functions of the form (32) is stable under quantum corrections. To see that this is generically the case note that the action (32) is formally invariant under the symmetry (31) provided one treats $\epsilon$ as a spurion field transforming as

$$
\epsilon \rightarrow \lambda^{-2} \epsilon
$$

Let us assume $\epsilon$ to be somewhat smaller than unity, so that one can perform perturbation theory in this parameter. Then the general form (32) of the action is invariant under quantum corrections whenever expansions in $\epsilon$ works (i.e., no terms proportional to negative powers of $\epsilon$ appear due to quantum corrections).

The models with the function $F$ obeying Eq. (30) have an interesting feature which is a consequence of the symmetry (31). It is straightforward to check that Eq. (31) implies the following relations among graviton masses in the Minkowski space,

$$
m_{0}^{2}=-3 \gamma m_{4}^{2}, \quad \gamma\left(m_{2}^{2}-3 m_{3}^{2}\right)=m_{4}^{2} .
$$

These relations ensure that the parameter $\mu^{2}$ defined by Eq. (23) is zero, i.e., the correction to the Newtonian potential [the last term in Eq. (22)] vanishes. Thus, barring the effects of the higher-derivative terms, at late times the only modification of gravity at the linear level is the nonzero mass of the two polarizations of the graviton. This suggests that the confining term in the Newtonian potential is unlikely to have any effect at present epoch. Indeed, the expressions for the graviton masses given in the Appendix A imply that the correction to the Newtonian potential goes to zero as $1 / a^{2 \nu_{m}}$, where $\nu_{m}$ is a minimal value of $\nu$ in Eq. (32). This parameter has to be fine-tuned to an extremely small value to allow for a substantial value of $\mu^{2}$ at present.

A particularly simple case occurs when the function $F$ depends only on the combination $Z=X^{\gamma} W^{i j}$. If $\gamma>1 / 3$ or $\gamma<0$, the evolution drives the system to the point $F_{Z}=$ 0 , in full similarity with the ghost condensate model. In the case $0<\gamma<1 / 3$ and regular $F, Z$ has to diverge at large 
$a$. This breaks the validity of the low-energy effective theory.

There are three boundary values of $\gamma$, which are somewhat special, namely $\gamma=1 / 3,0, \infty$. If $\gamma=1 / 3$ then $Z$ is constant during cosmological evolution and both $\rho_{1}$ and $\rho_{2}$ behave like a cosmological constant. An interesting property of this model is that a (constant) acceleration rate of the cosmological expansion is determined by the initial conditions in the Goldstone sector (the value of $Z$ ) rather than by the parameters of the action.

If $\gamma=0$ then $F(X, W)$ does not depend on $X$ at all. In this case Eq. (27) is satisfied automatically, and the only unconventional component in the Friedmann Eq. (26) is the last term $\rho_{2}$. This term may describe arbitrary equation of state depending on the choice of the function $F_{0}(W)$. For functions $F$ regular when $W$ goes to zero, this term becomes a cosmological constant as before.

In the case $\gamma=\infty$ the function $F$ depends on the scalar quantities $X, \operatorname{Tr} W^{2} /(\operatorname{Tr} W)^{2}$ and $\operatorname{Tr} W^{3} /(\operatorname{Tr} W)^{3}$. Flat cosmological solutions in such a theory have the same properties as in the ghost condensate model where the $F$ is a function of $X$ only. These theories, however, differ from the ghost condensate model in that they describe massive gravitons, and have different solutions in a nonflat case.

It is worth commenting on the role of the regular Minkowski vacua which are the points in the $(X, W)$ space at finite (nonzero) values of $X$ and $W$ where the energymomentum tensor of matter and Goldstone fields is zero and thus the Minkowski metric solves the Einstein equations. In the absence of matter, $\rho_{m}=0$, these points are determined by Eqs. (A10) and (A11). There may exist solutions to Eqs. (26) and (27) which asymptotically approach these points. These solutions correspond to the scale factor going to a finite limit, so they do not describe the current phase of the cosmological expansion.

To conclude this section we would like to stress that our analysis may not exhaust all viable cosmological solutions in the model with the action (4). For instance, the combination $X^{\gamma} / a^{2}$ may be proportional to some power of $\log a$ at late times, which is the case not covered above. Another possibility is to consider more general cosmological ansatz than that given by Eq. (24). Namely, one may consider the time-dependent configuration ${ }^{3}$ of the fields $\phi^{i}$,

$$
\phi^{i}=\Lambda^{2} C(t) x^{i}
$$

where $C(t)$ is an arbitrary function of time. Because of the symmetry (2) this ansatz is still homogeneous as the constant shift of the spatial coordinates $x^{i}$ can be compensated by the $\phi^{0}$-dependent shift of the fields $\phi^{i}$. The equations of motion on this ansatz reduce to two equations: the Friedmann Eq. (26) which remains unchanged, and the equation for $\phi^{0}$,

\footnotetext{
${ }^{3}$ We thank S. Sibiryakov for pointing out to us this possibility.
}

$$
\frac{X^{1 / 2}}{a^{3}} \partial_{t}\left(a^{3} X^{1 / 2} F_{X}\right)+3 \frac{\dot{C}}{C} W F_{W}=0 .
$$

For any fixed function $C(t)$, Eqs. (26) and (34) determine the dependence of $X$ and $a$ on time. Interestingly, in the case when the function $F$ is invariant under the additional dilatation symmetry (31), Eq. (34) takes the form (29) irrespectively of the particular shape of $C(t)$. Thus, while the time dependences of $X$ and $W$ separately vary with the choice $C(t)$, the evolution of $Z$ and the scale factor $a$ is universal. Consequently, observable quantities such as the expansion rate and the graviton masses do not depend on the function $C(t)$ if the symmetry (31) holds.

The situation is different if the dilatation symmetry is absent: in general, the expansion rate depends on the choice of the function $C(t)$. This ambiguity is a consequence of the symmetry (2) and is related to the presence of modes with the dispersion relation $p_{i}^{2}=0$. In order to fix this ambiguity one should specify boundary conditions for the fields $\phi^{i}$ at spatial infinity. To see this, imagine that the space is compact. For instance, if the space is a torus of the size $L$, the fields $\phi^{i}$ have to satisfy some kind of (quasi)periodicity condition, e.g.

$$
\phi^{i}\left(x^{i}\right)=\phi^{i}\left(x^{i}+L\right)-\Lambda^{2} L .
$$

This condition implies $C=$ const. Other boundary conditions may lead to time-dependent $C(t)$. In this sense, the ambiguity in choosing different functions $C(t)$ is analogous to the ambiguity in choosing the vacuum state in theories with flat directions.

\section{STABILITY}

Let us discuss the stability of the cosmological solutions obtained in the previous section. One should distinguish two different types of instabilities which may occur in a theory which is perturbatively stable about the flat background when the latter becomes curved. The first type of instabilities has the characteristic wavelength and timescales much longer that the inverse cutoff scale $\Lambda^{-1}$. They are set either by the curvature of the background, or suppressed by the powers of $\Lambda / M_{\mathrm{Pl}}$, if these instabilities appear due to mixing of higher-derivative terms with gravity (the latter type of instability is present, e.g., in the ghost condensate). We call these the infrared (IR) instabilities. Depending on a particular situation, the IR instabilities, if present, may be either dangerous or interesting phenomenologically (like, e.g., the Jeans instability). Their analysis is clearly important for the conclusion on the phenomenological viability of the model. However, even if present, the IR instabilities do not question the applicability of the analysis based on the low-energy effective field theory. We do not address IR stability of our models in the present paper.

The instabilities of a different type, which we refer to as ultraviolet (UV) instabilities, are those which occur at 
wavelengths (and/or time scales) much shorter than that of the background curvature, approaching the scales of order $\Lambda^{-1}$. Such instabilities do affect the structure of the theory in the ultraviolet and imply the breakdown of the effective field theory description for scales much lower than $\Lambda$. An example of such an instability is the Boulware-Deser instability [6] which occurs in the curved background in the Fierz-Pauli theory of massive gravity due to the presence of the ghost mode. ${ }^{4}$ We will see that the instabilities of this type are absent in our models. A physical reason is that massive gravities with symmetry (2) can be thought of as stable scalar theories coupled to the Einstein gravity, which is not possible in the Fierz-Pauli case.

The origin of the Boulware-Deser instability is easy to understand within the formalism of the Goldstone fields $\phi^{\mu}$. The Goldstone action which corresponds to a Lorentzinvariant massive gravity, including the Fierz-Pauli theory, is [cf. the second term in Eq. (4)]

$$
S_{G}=\Lambda^{4} \int \sqrt{-g} d^{4} x F(P),
$$

where $P=g^{\mu \nu} \eta_{\alpha \beta} \partial_{\mu} \phi^{\alpha} \partial_{\nu} \phi^{\beta}$ and $\eta_{\alpha \beta}$ is a Minkowski metric. In the Minkowski background (6), the quadratic action for the Goldstone perturbations $\delta \phi^{\mu} \equiv \xi^{\mu}$ takes the form

$$
L=\mu_{1}^{2}(P)\left(\partial_{\mu} \xi^{\alpha}\right)^{2}+\mu_{2}^{2}(P)\left(\partial_{\mu} \xi^{\mu}\right)^{2},
$$

where the coefficients $\mu_{i}^{2}(P)$ are some functions of $P=$ $\alpha^{2}-3 \beta^{2}$ which are expressed in terms of the first and second derivatives of the function $F(P)$. The particular expressions are irrelevant for the argument; what is important is the fact that the coefficients $\mu_{i}^{2}(P)$ do depend on $P$. The Lagrangian (36) describes the ghost-free theory only in the case

$$
\mu_{1}^{2}(P)+\mu_{2}^{2}(P)=0,
$$

when it is proportional to $\left(\partial_{\mu} \xi_{\nu}-\partial_{\nu} \xi_{\mu}\right)^{2}$. In general, this condition is satisfied in an isolated point $P=P_{0}$.

To see the instability, consider now the perturbations localized in the vicinity of a given point in the background of some nontrivial solution. In the UV limit the metric can be approximated as flat, so the perturbations in the Goldstone sector will be described by the Lagrangian (36). However, since Goldstone fields depend on space

\footnotetext{
${ }^{4}$ Note, however, that the statement [16] that rapid classical instabilities are present in the Fierz-Pauli theory in the Minkowski background is unjustified. This claim is based on the analysis of the spatially homogeneous solutions, while to address the issue of stability one should study dynamics of the spatially localized excitations of finite energy. An example illustrating this point is provided, e.g., by the massless scalar field with the negative potential $V=-\lambda \phi^{4}$. From the analysis of the spatially homogeneous solutions one might conclude that vacuum $\phi=0$ is perturbatively unstable in this theory, which is not the case (see, e.g. [17]).
}

and time, the condition (37) will not, in general, be satisfied (because, for instance, the value of $P$ is time dependent for cosmological solutions). The Fierz-Pauli theory is therefore UV unstable in a curved background even if this background is locally very close to the Minkowski one. This implies that the cutoff scale of the low-energy effective theory in the curved backgrounds generically is even lower than in the Minkowski background, as it should be smaller than a mass of the ghost mode. A detailed discussion of the corresponding scales in the phenomenologically relevant backgrounds can be found in [18].

Let us now repeat the same analysis for our model and show that it is free from UV instabilities at least for backgrounds which are close to the vacuum (6) in the UV limit. For simplicity, consider the model obeying the dilatation symmetry (31). The Goldstone action has the form

$$
S_{G}=\Lambda^{4} \int \sqrt{-} g d^{4} x F\left(Z^{i j}\right),
$$

where $Z^{i j}=X^{\gamma} W^{i j}$, and the quantities $X$ and $W^{i j}$ are given by Eq. (5). Deep in the UV region where the metric can be considered as flat, any Goldstone configuration of the form (6) is a solution to the Goldstone field equations ${ }^{5}$. In this background, the variable $Z^{i j}$ takes the values depending on the constants $\alpha$ and $\beta$. The quadratic Lagrangian for the Goldstone perturbations $\xi^{0}$ and $\xi^{i}$ reads

$$
\begin{aligned}
L= & M_{\mathrm{Pl}}^{2}\left\{2 m_{0}^{2}\left(\partial_{0} \xi_{0}\right)^{2}+m_{1}^{2}\left(\partial_{i} \xi_{0}\right)^{2}+4 m_{4}^{2} \xi_{0} \partial_{0} \partial_{i} \xi_{i}\right. \\
& \left.-m_{2}^{2}\left(\partial_{i} \xi_{j}\right)^{2}-\left(m_{2}^{2}-2 m_{3}^{2}\right)\left(\partial_{i} \xi_{i}\right)^{2}\right\},
\end{aligned}
$$

where the kinetic coefficients $m_{i}$ are certain functions of $Z^{i j}$ (and therefore, of $\alpha$ and $\beta$ ). Their explicit expressions are given in the Appendix B. Using these expressions one can check that the kinetic coefficients satisfy the constraints

$$
m_{0}^{2}=-3 \gamma m_{4}^{2}, \quad \gamma\left(m_{2}^{2}-3 m_{3}^{2}\right)=m_{4}^{2}-\frac{1}{2} m_{1}^{2},
$$

which follow from the symmetry (2). Note that these constraints differ from Eqs. (33) because the background we consider now does not correspond to the zero energymomentum tensor of the Goldstone fields. They reproduce Eqs. (33) at $m_{1}^{2}=0$.

It is convenient to decompose the Goldstone perturbations into the transverse vector $\xi_{i}^{T}\left(\partial^{i} \xi_{i}^{T}=0\right)$ and two scalars $\xi_{L}$ and $\xi_{0}$, as defined by the following relation:

$$
\xi_{i}=\xi_{i}^{T}+\frac{1}{\sqrt{-\partial_{i}^{2}}} \partial_{i} \xi_{L} .
$$

The Lagrangian for the vector part reads

\footnotetext{
${ }^{5}$ In principle, one may consider a larger class of backgrounds, e.g. those with $\phi^{i}=B_{j}^{i} x^{j}$. For definiteness, we restrict our discussion to rotationally invariant case. This choice covers, in particular, cosmological solutions obtained above.
} 


$$
L=-M_{\mathrm{Pl}}^{2} m_{2}^{2}\left(\partial_{i} \xi_{j}^{T}\right)^{2} .
$$

Both modes in the vector sector have the dispersion relation $p_{i}^{2}=0$ and do not propagate, independently of the values of $\alpha$ and $\beta$; this is a consequence of the symmetry (2). There are no instabilities in this sector.

The Lagrangian for scalar perturbations $\xi_{0}$ and $\xi_{L}$ is

$$
\begin{aligned}
L= & M_{\mathrm{Pl}}^{2}\left\{2 m_{0}^{2}\left(\partial_{0} \xi_{0}\right)^{2}+m_{1}^{2}\left(\partial_{i} \xi_{0}\right)^{2}-4 m_{4}^{2} \xi_{0} \partial_{0} \sqrt{-\partial_{i}^{2}} \xi_{L}\right. \\
& \left.-2\left(m_{2}^{2}-m_{3}^{2}\right)\left(\partial_{i} \xi_{L}\right)^{2}\right\} .
\end{aligned}
$$

In the Fourier space it can be written as

$$
L=M_{\mathrm{Pl}}^{2} \cdot \xi^{\dagger} M \xi,
$$

where $\xi=\left(\xi_{0}, \xi_{L}\right)$ and the $2 \times 2$ matrix $M$ has the form

$$
M=\left(\begin{array}{cc}
2 m_{0}^{2} \omega^{2}+m_{1}^{2} p^{2} & -2 i m_{4}^{2} \omega p \\
2 i m_{4}^{2} \omega p & 2\left(m_{3}^{2}-m_{2}^{2}\right) p^{2}
\end{array}\right)
$$

with $p=\sqrt{p_{i}^{2}}$. The eigenvectors of the matrix $M$ correspond to physical excitations. The eigenvalues can be written as

$$
M_{ \pm}=\frac{1}{2}\left\{T \pm \sqrt{T^{2}-4 D}\right\},
$$

where $T=\operatorname{Tr}(M)$ and $D=\operatorname{det}(\mathrm{M})$. They determine two dispersion relations $\omega_{ \pm}^{2}\left(p^{2}\right)$ by the implicit equations

$$
M_{ \pm}\left(\omega^{2}, p^{2}\right)=0 .
$$

The system is classically stable if $\omega_{ \pm}^{2}\left(p^{2}\right)>0$ for all relevant $p^{2}$. The system has no ghosts if near the mass shell the terms linear in $\omega^{2}$ are nonnegative,

$$
\left.\frac{\partial M_{ \pm}\left(\omega^{2}, p^{2}\right)}{\partial \omega^{2}}\right|_{\omega^{2}=\omega_{ \pm}^{2}\left(p^{2}\right)} \geq 0,
$$

for both modes.

The mode which corresponds to the eigenvalue $M_{-}$has the dispersion relation $p^{2}=0$ and does not propagate. The inequality (44) is marginally satisfied, so this mode does not cause the UV instability. Note that the existence of the scalar mode with the dispersion relation $p^{2}=0$ is guaranteed by the reparametrization symmetry (2).

It is worth mentioning a physical interpretation of the modes with the dispersion relation $p^{2}=0$. They can be thought of as degrees of freedom with infinite propagation velocity (unlike the ghost condensate mode which has zero velocity at zero-derivative level and acquires a very small velocity due to higher-derivative terms). Physically, they describe sound waves propagating through the rigid coordinate frame selected in space by the functions $\phi^{i}$. The rigidity of this frame is ensured by the symmetry (2) and $S O(3)$ symmetry of the Goldstone action that allow to move and rotate this frame only as a whole. Note that infinitely fast propagating modes do not imply the violation of causality in the absence of Lorentz invariance, but allow for instantaneous transfer of information. A recent discussion of some of the properties of these modes in the toy QED model can be found in Refs. [15,19].

The mode which corresponds to the eigenvalue $M_{+}$has the dispersion relation

$$
\omega^{2}=v^{2} p^{2}
$$

where

$$
v^{2}=\frac{1}{2} \frac{m_{1}^{2}\left(m_{3}^{2}-m_{2}^{2}\right)}{m_{4}^{4}-m_{0}^{2}\left(m_{3}^{2}-m_{2}^{2}\right)} .
$$

The absence of classical instabilities thus requires

$$
\frac{m_{1}^{2}\left(m_{3}^{2}-m_{2}^{2}\right)}{m_{4}^{4}-m_{0}^{2}\left(m_{3}^{2}-m_{2}^{2}\right)}>0 \text {. }
$$

When this condition is satisfied, Eq. (44) which ensures the absence of ghosts translates into the following inequality (see Appendix B for details),

$$
m_{0}^{2}-\frac{m_{4}^{4}}{m_{3}^{2}-m_{2}^{2}}>0
$$

in agreement with the result of Ref. [3]. Thus, there are neither classical instabilities nor ghosts in our model provided that both conditions (46) and (47) are satisfied. These conditions are compatible with the constraints (40).

For a flat background $m_{1}^{2}=0$, so one may worry about UV stability of an arbitrarily close background with the positive value of $m_{1}^{2}$ and, consequently, negative velocity $v^{2}<0$. In the vicinity of the point where $v^{2}=0$ the higher-derivative terms in the dispersion relation become important, so that it takes the form $\omega^{2}=v^{2} p^{2}+\alpha p^{4} / \Lambda^{2}$, where $\alpha$ is a coefficient of order one which we assume to be positive. It is clear now that close to the point $v^{2}=0$ the instability occurs only at very low momenta, i.e., in the IR region. The situation here is the same as in the ghost condensate model. By analogy we expect that accounting for mixing with gravity for higher-derivative terms leads to the IR instability of this type already in the flat background with $m_{1}^{2}=0$.

The case $\gamma=0$ when the Goldstone action depends only on $W^{i j}$ requires a separate consideration. Using the expressions for the graviton masses given in the Appendix A, Eqs. (A13)-(A17), one finds that in this case $m_{0}^{2}=m_{1}^{2}=$ $m_{4}^{2}=0$ in the Minkowski background. Therefore, this is a theory with $\Delta=0$ discussed in the end of Sec. II. At the one-derivative level this theory possesses a symmetry $\phi^{0} \rightarrow \phi^{0}+\xi^{0}\left(\phi^{0}\right)$ apart from the symmetry $\phi^{i} \rightarrow \phi^{i}+$ $\xi^{i}\left(\phi^{0}\right)$. (This symmetry should be imposed at the higherderivative level in order to avoid ghosts.) Naively, one may expect that the above symmetries imply that all modes should always have the dispersion relation $p^{2}=0$. However, the situation is more subtle. From Eq. (B1) of Appendix B one finds that in the curved background only the mass $m_{0}^{2}$ is equal to zero, while $m_{1}^{2}$ and $m_{4}^{2}$ may be 
nonzero. As a result, in curved backgrounds, in addition to two solutions with $p^{2}=0, \xi_{L}(t)$ and $\xi_{0}(t)$, there is also a mode with the velocity

$$
v^{2}=\frac{1}{2} \frac{m_{1}^{2}\left(m_{3}^{2}-m_{2}^{2}\right)}{m_{4}^{4}}
$$

which is very large for backgrounds close to the Minkowski one because $m_{1}^{2} \sim m_{4}^{2}$ are both very small. Of these three modes, only one $\left(\xi_{L}(t)\right)$ is seen in the quadratic action about flat background at the one-derivative level. We believe that further analysis is needed to understand whether the two new modes lead to the problems like low strong-coupling scale. Note, however, that unlike in the Fierz-Pauli case, the new modes are not ghosts, provided the condition (47) holds.

We see that the situation in our models is quite different from that in the Fierz-Pauli theory of massive gravity. Unlike the latter, our models are free of ghosts in a finite region of coefficients $m_{a}^{2}$ in Eq. (39) (and, therefore, of constants $\alpha$ and $\beta$ ) which includes the point corresponding to the flat background with $m_{1}^{2}=0$. Thus, with a proper choice of the function $F$ and higher-derivative terms, our models are UV stable at least for backgrounds close to the flat one. The Boulware-Deser instability is absent.

\section{DISCUSSION}

In order to be more than a theoretical exercise, the theory of Lorentz-violating massive gravity must eventually address the fundamental puzzles of modern cosmology such as the origin of dark matter and dark energy. The class of models discussed in this paper provides a number of possibilities in this direction.

As follows from Sec. III, the evolution of the Universe may naturally lead to the attractor which corresponds to the theory possessing the dilatation symmetry (31). In this case, the relations (33) among masses imply that the growing term in the Newtonian potential vanishes. Even in this simplest version, the model has a number of features interesting from the cosmological and observational points of view. First, the massive graviton itself is a candidate for the dark matter particle [13]. This possibility is observationally testable, the current limits being plotted in Fig. 1. The constraints will be improved in the near future by the data from the Australian pulsar timing array [20]. If massive gravitons do not constitute all of the dark matter, they are still detectable in a certain range of masses because they would produce a unique monochromatic signal in gravitational wave detectors such as LISA [21].

Second, the Goldstone fields give two extra contributions to the Friedmann Eq. (26) which we denoted $\rho_{1}$ and $\rho_{2}$ in Sec. III. For $1 / 3<\gamma<1$ the first of these contributions $\rho_{1}$ behaves like a "quintessence" with the equation of state varying from $w=-1$ to $w=-1 / 3$ for different values of the parameter $\gamma$, which characterizes the dilatation symmetry emerging in the cosmological attractor [see

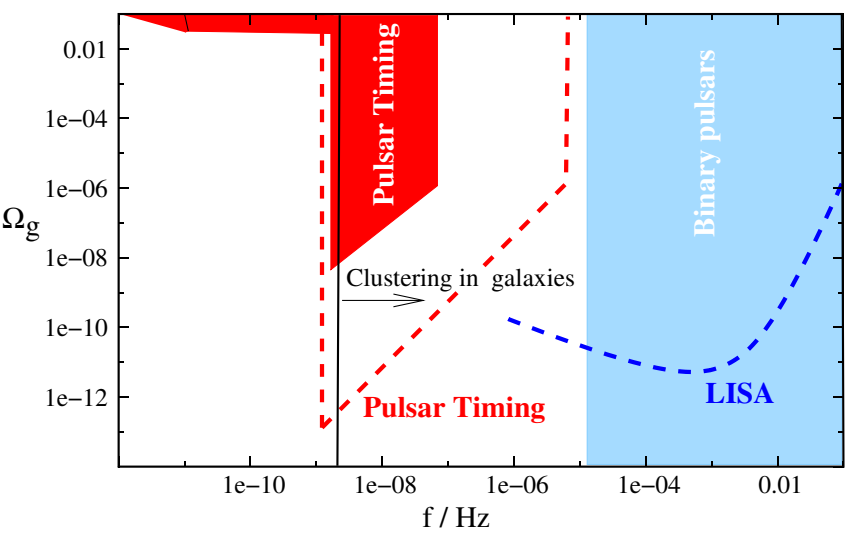

FIG. 1 (color online). Limits on the gravitational wave signal in the frequency/relative graviton abundance plane. Light shaded region is excluded by the observations of binary pulsars [23]. Dark shaded region is excluded by the timing of the millisecond pulsars [29]. Dashed lines show the expected sensitivity of the Australian pulsar timing array and LISA. Frequencies higher than that marked by the solid line correspond to graviton masses large enough to allow for gravitons to cluster in galaxies. Note that if all of the galactic dark matter is comprised of massive gravitons then the gravitational wave signal corresponds to graviton abundance $\Omega_{g} \sim 10^{5}$.

Eq. (33)]. The second contribution $\rho_{2}$ behaves as a cosmological constant.

An interesting special situation takes place for $\gamma=1 / 3$. In this case both contributions $\rho_{1}$ and $\rho_{2}$ have vacuum equation of state $\omega=-1$. As a result the acceleration rate of the late de Sitter phase is a dynamical quantity, determined by the initial conditions in the Goldstone sector rather than by parameters of the action. This is similar to situation in the unimodular gravity ${ }^{6}$ [22], where cosmological constant is also a constant of integration. This similarity appears to be not just a coincidence. The metric determinant $g$ is invariant under the symmetry (2) and under the dilatation symmetry (31) with $\gamma=1 / 3$, so the symmetry group of massive gravity is a subgroup of the unimodular gravity in this case.

On the other hand there is an important difference between massive gravity with $\gamma=1 / 3$ and unimodular gravity. Namely, the only difference between unimodular gravity and the Einstein theory (at least at the classical level) is that in the former case solutions with arbitrary values of cosmological constant are present independently of the value of the vacuum energy. On the contrary, in massive gravity the contribution of the Goldstone sector has the form of a cosmological constant only for flat homogeneous cosmological solutions. In particular, the initial conditions in the Goldstone sector may vary in space resulting in solutions with different values of the acceleration rate in different parts of the Universe. Such solutions

\footnotetext{
${ }^{6}$ We thank John March-Russel for pointing out this similarity.
} 
are absent in both general relativity and unimodular gravity. If nothing else, this allows the application of the anthropic arguments [22]. There is a caveat, however. In order to cancel a bare cosmological constant that is much larger than $\Lambda^{4}$ (where $\Lambda$ is the cutoff scale of our model) one needs a fine-tuning to keep the mass of the graviton from being too large. It is not impossible to imagine an anthropic explanation for this fine-tuning as well-according to the estimates of Ref. [13] relic massive gravitons with masses higher than $\left(10^{15} \mathrm{~cm}\right)^{-1}$ (upper bound from the timing of the binary pulsars [23]) are likely to overclose the Universe. Alternatively, one may hope that the unusual properties of cosmological solutions in massive gravity may be a first step towards a dynamical solution of the dark energy problem.

The situation may become more complicated if the model is kept away from the dilatation-symmetric attractor by a fine-tuning of the cosmological evolution or some other mechanism (e.g., if $X^{\gamma} / a^{2}$ is proportional to some power of $\log a$ at late times, instead of being a constant). Then the potential of a pointlike source acquires - formally - the linearly growing contribution, Eq. (3). There are two distance scales associated with this contribution. The first one, $l_{1} \sim 1 / m$, determines the distances where the growing term starts to dominate over the conventional one. The second scale is $l_{2}=\left(M_{\mathrm{Pl}}^{2} / \mathrm{Mm}^{2}\right)$; it depends on the mass $M$ of the source. At distance $l_{2}$ the potential $\Phi$ becomes of order unity indicating the breakdown of perturbation theory and possible onset of a nonlinear regime. Note that in the gauge we are using, the metric components that become large are

$$
h_{0 i} \sim n_{i} \frac{t}{l_{2}},
$$

where $n_{i}$ is a unit vector in the direction of the source. Consequently, nonlinear regime starts at the moment of time $t_{2} \sim l_{2}$ rather than at a certain distance from the source.

To understand this qualitatively, recall that our choice of the integration constant $\psi_{0}\left(x^{i}\right)=0$ corresponds to the initially homogeneous Universe. One can view the Goldstone sector as a (multicomponent) fluid which is accreted by sources after they are formed. Eventually, this accretion results in the onset of the nonlinear regime; qualitatively, this happens at the time of order $l_{2}$ after the formation of sources. It is tempting to speculate that this nonlinear phase may result in the nonzero profile of the ghost condensate mode $\psi_{0}$ such that the linearly growing term in the potential (21) is canceled. The corresponding nonlinear dynamics is presumably similar to that of the ghost condensate model and is not sufficiently understood at the moment (see [24-26] for some proposals in this direction). One characteristic feature of the ghost condensate dynamics is the presence of strong retardation effects $[1,27,28]$, so one may think that the cancellation is incom- plete, leading to the logarithmically growing potential needed to explain flat rotation curves. Note that nonlinear effects related to the ghost condensate mode are present even when linearly growing terms in the potential are forbidden by dilatation symmetries, so understanding of these effects is one of the most pressing questions for this kind of models.

\section{ACKNOWLEDGMENTS}

We thank Nima Arkani-Hamed, Thomas Gregoire, Markus Luty, John March-Russel, Alberto Nicolis, Riccardo Rattazzi, Valery Rubakov, Mikhail Sazhin, Matt Schwartz, and Sergei Sibiryakov for useful discussions and correspondence. S. D. thanks Harvard Theory Group where part of this work was done for a warm hospitality. The work of P.T. is supported by IISN, Belgian Science Policy (under Contract No. IAP V/27).

\section{APPENDIX A}

In this appendix we calculate mass terms of the gravitational field in the Friedmann background. The mass terms come from the expansion of the second term in the rhs of Eq. (4) in powers of the perturbation $\delta g_{\mu \nu}$ to the quadratic order about the background metric

$$
d s^{2}=g_{\mu \nu} d x^{\mu} d x^{\nu}=a^{2}(\eta)\left(d \eta^{2}-d \mathbf{x}^{2}\right) .
$$

With the definitions (5) one has

$$
\begin{aligned}
\sqrt{-(g+\delta g)}= & a^{4}+\frac{a^{2}}{2}\left(\delta g_{00}-\delta g_{i i}\right)-\frac{1}{8} \delta g_{00}^{2} \\
& -\frac{1}{4} \delta g_{00} \delta g_{i i}+\frac{1}{8} \delta g_{i i}^{2}+\frac{1}{2} \delta g_{0 i}^{2}-\frac{1}{4} \delta g_{i j}^{2} \\
& +\ldots, \\
X(g+\delta g)= & X(g)\left[1-\frac{1}{a^{2}} \delta g_{00}+\frac{1}{a^{4}}\left(\delta g_{00}^{2}-\delta g_{0 i}^{2}\right)\right. \\
& +\ldots], \\
W^{i j}(g+\delta g)= & W(g)\left[-\delta_{i j}-\frac{1}{a^{2}} \delta g_{i j}-\frac{1}{a^{4}} \delta g_{i k} \delta g_{k j}\right. \\
& +\ldots]
\end{aligned}
$$

where

$$
W \equiv-\frac{1}{3} \delta_{i j} W^{i j} .
$$

Because of the rotational symmetry, the derivatives of $F$ up to the second order are expressed in terms of the 6 scalar quantities $F_{X}, F_{W}, F_{X X}, F_{X W}, F_{W W 1}$ and $F_{W W 2}$ which are defined as follows:

$$
\frac{\partial}{\partial X} F\left(X, W^{i j}\right)=F_{X},
$$




$$
\begin{gathered}
\frac{\partial}{\partial W^{i j}} F\left(X, W^{i j}\right)=F_{W} \delta_{i j}, \\
\frac{\partial^{2}}{\partial X^{2}} F\left(X, W^{i j}\right)=F_{X X}, \\
\frac{\partial^{2}}{\partial X \partial W^{i j}} F\left(X, W^{i j}\right)=F_{X W} \delta_{i j}, \\
\frac{\partial^{2} \quad}{\partial W^{i j} \partial W^{m n}} F\left(X, W^{i j}\right)=F_{W W 1} \delta_{i j} \delta_{m n} \\
+F_{W W 2}\left(\delta_{i m} \delta_{j n}+\delta_{i n} \delta_{j m}\right) .
\end{gathered}
$$

The derivatives on the left-hand side of these equations are all evaluated at the point $X(g), W^{i j}(g)$.

With these definitions, the linear contribution to the expansion of the second term in Eq. (4) is

$$
a^{2}\left(\frac{1}{2} F-X F_{X}\right) \delta g_{00}-a^{2}\left(\frac{1}{2} F+W F_{W}\right) \delta g_{i i} .
$$

The corresponding Friedmann equations are

$$
\begin{gathered}
\frac{3 a^{\prime 2}}{a^{4}}=8 \pi G\left[\Lambda^{4}\left(2 X F_{X}-F\right)+\rho_{m}\right], \\
\frac{2 a^{\prime \prime}}{a^{3}}-\frac{a^{\prime 2}}{a^{4}}=-8 \pi G\left[\Lambda^{4}\left(2 W F_{W}+F\right)+p_{m}\right],
\end{gathered}
$$

where $\rho_{m}, p_{m}$ are the energy density and pressure of matter. Combination of these two equations gives the equation of motion for the field $\phi^{0}$,

$$
a^{3} \sqrt{X} F_{X}=\text { const. }
$$

In the Minkowski background one has

$$
\begin{gathered}
F-2 X F_{X}=0, \\
F+2 W F_{W}=0 .
\end{gathered}
$$

For a generic function $F$ these equations are satisfied for some $X, W$.

The quadratic part of the Lagrangian Eq. (4) with respect to metric perturbations about Friedmann background is

$$
\begin{aligned}
\Lambda^{4} & \left\{\frac{1}{2} X^{2} F_{X X}+\frac{1}{2} X F_{X}-\frac{1}{8} F\right\} \delta g_{00}^{2}+\Lambda^{4}\left\{\frac{1}{2} F-X F_{X}\right\} \delta g_{0 i}^{2} \\
& +\Lambda^{4}\left\{X W F_{X W}-\frac{1}{2} W F_{W}+\frac{1}{2} X F_{X}-\frac{1}{4} F\right\} \delta g_{00} \delta g_{i i} \\
& +\Lambda^{4}\left\{\frac{1}{2} W^{2} F_{W W 1}+\frac{1}{2} W F_{W}+\frac{1}{8} F\right\} \delta g_{i i}^{2} \\
& +\Lambda^{4}\left\{W^{2} F_{W W 2}-W F_{W}-\frac{1}{4} F\right\} \delta g_{i j}^{2},
\end{aligned}
$$

Comparing this expression to Eq. (10) one finds for the masses of the gravitational field in the Minkowski vacuum,

$$
\begin{gathered}
m_{0}^{2}=\frac{\Lambda^{4}}{M_{\mathrm{Pl}}^{2}}\left\{X F_{X}+2 X^{2} F_{X X}\right\}, \\
m_{1}^{2}=0, \\
m_{2}^{2}=-\frac{\Lambda^{4}}{M_{\mathrm{Pl}}^{2}}\left\{2 X F_{X}+4 W^{2} F_{W W 2}\right\}, \\
m_{3}^{2}=\frac{\Lambda^{4}}{M_{\mathrm{Pl}}^{2}}\left\{-X F_{X}+2 W^{2} F_{W W 1}\right\}, \\
m_{4}^{2}=-\frac{\Lambda^{4}}{M_{\mathrm{Pl}}^{2}}\left\{X F_{X}+2 X W F_{X W}\right\} .
\end{gathered}
$$

\section{APPENDIX B}

In this appendix we provide some intermediate formulas skipped in Sec. IV.

The explicit expressions for the kinetic coefficients in the action (39) are

$$
\begin{aligned}
m_{0}^{2}= & -\frac{6 \Lambda^{4}}{M_{P l}^{2}}\left\{\gamma\left(\gamma-\frac{1}{2}\right) F_{Z} Z-3 \gamma^{2} F_{Z Z 1} Z^{2}\right. \\
& \left.-2 \gamma^{2} F_{Z Z 2} Z^{2}\right\}, \\
m_{1}^{2}= & \frac{2 \Lambda^{4}}{M_{P l}^{2}}(3 \gamma-1) F_{Z} Z, \\
m_{2}^{2}= & \frac{2 \Lambda^{4}}{M_{\mathrm{Pl}}^{2}}\left\{F_{Z} Z-2 F_{Z Z 2} Z^{2}\right\}, \\
m_{3}^{2}= & \frac{2 \Lambda^{4}}{M_{P l}^{2}}\left\{\frac{1}{2} F_{Z} Z+F_{Z Z 1} Z^{2}\right\}, \\
m_{4}^{2}= & \frac{2 \Lambda^{4}}{M_{\mathrm{Pl}}^{2}}\left\{\left(\gamma-\frac{1}{2}\right) F_{Z} Z-3 \gamma F_{Z Z 1} Z^{2}-2 \gamma F_{Z Z 2} Z^{2}\right\} .
\end{aligned}
$$

Here $Z \equiv-Z^{i j} \delta_{i j} / 3$, while the scalar functions $F_{Z}, F_{Z Z 1}$ and $F_{Z Z 2}$ are defined by the following relations:

$$
\begin{aligned}
\frac{\partial}{\partial Z^{i j}} F\left(Z^{i j}\right) & =F_{Z} \delta_{i j}, \\
\frac{\partial^{2}}{\partial Z^{i j} \partial Z^{m n}} F\left(Z^{i j}\right) & =F_{Z Z 1} \delta_{i j} \delta_{m n}+F_{Z Z 2}\left(\delta_{i m} \delta_{j n}+\delta_{i n} \delta_{j m}\right) .
\end{aligned}
$$

It is straightforward to check that coefficients (B1) satisfy relations (40).

Calculation of the no-ghost condition (47) proceeds as follows:

$$
\left.\frac{\partial M_{ \pm}\left(\omega^{2}, p^{2}\right)}{\partial \omega^{2}}\right|_{\omega^{2}=\omega_{ \pm}^{2}\left(p^{2}\right)}=\frac{1}{2}\left(T^{\prime} \pm \frac{T T^{\prime}-2 D^{\prime}}{|T|}\right)=\frac{D^{\prime}}{T},
$$


where prime denotes differentiation with respect to $\omega^{2}$, and in algebraic transformation we were taking into account, that we are taking the derivative of the eigenvalue which is zero on-shell. Plugging explicit expressions for $D$ and $T$, following from Eq. (43), we obtain the following condition for the propagating mode to be not a ghost

$$
\frac{m_{0}^{2}\left(m_{3}^{2}-m_{2}^{2}\right)-m_{4}^{4}}{2 m_{0}^{2} v^{2}+m_{1}^{2}+2\left(m_{3}^{2}-m_{2}^{2}\right)}>0
$$

where $v^{2}$ is given by Eq. (45). Using explicit expression (45) one can check that at $v^{2}>0$ this condition is equivalent to (47).
[1] N. Arkani-Hamed, H.C. Cheng, M. A. Luty, and S. Mukohyama, J. High Energy Phys. 05 (2004) 074.

[2] V. Rubakov, hep-th/0407104.

[3] S. L. Dubovsky, J. High Energy Phys. 10 (2004) 076.

[4] H. van Dam and M. J. G. Veltman, Nucl. Phys. B22, 397 (1970).

[5] V. I. Zakharov, JETP Lett. 12, 312 (1970).

[6] D. G. Boulware and S. Deser, Phys. Rev. D 6, 3368 (1972).

[7] N. Arkani-Hamed, H. Georgi, and M. D. Schwartz, Ann. Phys. (N.Y.) 305, 96 (2003).

[8] A. I. Vainshtein, Phys. Lett. B 39, 393 (1972).

[9] C. Deffayet, G. R. Dvali, G. Gabadadze, and A. I. Vainshtein, Phys. Rev. D 65, 044026 (2002).

[10] A. Nicolis and R. Rattazzi, J. High Energy Phys. 06 (2004) 059.

[11] T. Jacobson and D. Mattingly, Phys. Rev. D 64, 024028 (2001).

[12] B. M. Gripaios, J. High Energy Phys. 10 (2004) 069.

[13] S. L. Dubovsky, P. G. Tinyakov, and I. I. Tkachev, Phys. Rev. Lett. 94, 181102 (2005).

[14] V. F. Mukhanov, H. A. Feldman, and R. H. Brandenberger, Phys. Rep. 215, 203 (1992).
[15] G. Gabadadze and L. Grisa, Phys. Lett. B 617, 124 (2005).

[16] G. Gabadadze and A. Gruzinov, hep-th/0312074.

[17] D. T. Son and P. G. Tinyakov, Nucl. Phys. B415, 101 (1994).

[18] P. Creminelli, A. Nicolis, M. Papucci, and E. Trincherini (to be published).

[19] G. Dvali, M. Papucci, and M.D. Schwartz, Phys. Rev. Lett. 94, 191602 (2005).

[20] G. Hobbs, astro-ph/0412153.

[21] P. L. Bender, Classical Quantum Gravity 20, S301 (2003).

[22] S. Weinberg, Rev. Mod. Phys. 61, 1 (1989).

[23] J. H. Taylor, Rev. Mod. Phys. 66, 711 (1994).

[24] A. V. Frolov, Phys. Rev. D 70, 061501 (2004).

[25] D. Krotov, C. Rebbi, V. A. Rubakov, and V. Zakharov, Phys. Rev. D 71, 045014 (2005).

[26] S. Mukohyama, Phys. Rev. D 71, 104019 (2005).

[27] S. L. Dubovsky, J. Cosmol. Astropart. Phys. 07 (2004) 009.

[28] M. Peloso and L. Sorbo, Phys. Lett. B 593, 25 (2004).

[29] A. N. Lommen, astro-ph/0208572. 